\title{
At the Click of a Mouse: APSR's Most Downloaded Articles
}

What are the most frequently read articles in political science? the most cited? the most influential? the best? No one knows, and probably no one ever will. Even the much-referenced Social Science Citation Index provides only a trace of any particular journal article's legacy. Even if all the citations are counted, what does that mean?

Stopping just short of claiming that this ranking signifies nothing at all, here are the Top 25 American Political Science Review articles as measured by the number of times each has been accessed via JSTOR (www. jstor.org). Qualifications of these rankings include: only individuals at institutions with JSTOR subscriptions (about 450 colleges, universities, and research centers at last count) could download articles during the reporting period, a majority of the downloads were most likely done by students getting supplementary course readings, and viewings and printings do not equal readings, understandings, or influencings.

Beginning this year, individual APSA members can subscribe to JSTOR for APSR-only access when they renew their Association memberships. Subscriptions cost \$25, and are primarily recommended for members at institutions that do not currently subscribe to JSTOR.

\begin{tabular}{|c|c|c|c|}
\hline Rank & Article & Viewings & Printings \\
\hline 1 & Graham T. Allison. 1969. "Conceptual Models and the Cuban Missile Crisis." 63(Sep.): 689-718. & 1,055 & 713 \\
\hline 2 & $\begin{array}{l}\text { Stephen Ansolabehere, Shanto lyengar, Adam Simon, Nicholas Valentino. 1994. "Does Attack Advertising } \\
\text { Demobilize the Electorate?" 88(Dec.): 829-38. }\end{array}$ & 530 & 297 \\
\hline 3 & $\begin{array}{l}\text { Sidney Verba, Kay Lehman Scholzman, Henry Brady, Norman H. Nie. 1993. "Citizen Activity: Who } \\
\text { Participates? What Do They Say?" 87(Jun.): 303-18. }\end{array}$ & 476 & 319 \\
\hline 4 & $\begin{array}{l}\text { James G. March, Johan P. Olsen. 1984. "The New Institutionalism: Organizational Factors in Political } \\
\text { Life." 78(Sep): 734-49. }\end{array}$ & 395 & 400 \\
\hline 5 & $\begin{array}{l}\text { Rogers M. Smith. 1993. "Beyond Tocqueville, Myrdal, and Hartz: The Multiple Traditions in America." } \\
\text { 87(Sep.): 549-66. }\end{array}$ & 525 & 249 \\
\hline 6 & Larry M. Bartels. 1993. "Messages Received: The Political Impact of Media Exposure." 87(Jun.): 267-85. & 462 & 278 \\
\hline 7 & $\begin{array}{l}\text { Benjamin I. Page, Robert Y. Shapiro, Glenn R. Dempsey. 1987. "What Moves Public Opinion?" 81(Mar.): } \\
\text { 23-44. }\end{array}$ & 478 & 260 \\
\hline 8 & $\begin{array}{l}\text { Ross E. Burkhart, Michael S. Lewis-Beck. 1994. "Comparative Democracy: The Economic Development } \\
\text { Thesis." 88(Dec.): 903-10. }\end{array}$ & 508 & 206 \\
\hline 9 & Benjamin I. Page, Robert Y. Shapiro. 1983. "Effects of Public Opinion on Policy." 77(Mar.): 175-90. & 471 & 207 \\
\hline 10 & G. Bingham Powell Jr. 1986. "American Voter Turnout in Comparative Perspective." 80(Mar.): 17-43. & 392 & 262 \\
\hline 11 & $\begin{array}{l}\text { Seymour Martin Lipset. 1959. "Some Social Requisites of Democracy: Economic Development and } \\
\text { Political Legitimacy." 53(Mar.): 69-105. }\end{array}$ & 397 & 256 \\
\hline 12 & Michael W. Doyle. 1986. "Liberalism and World Politics." 80(4): 1151-69. & 418 & 233 \\
\hline 13 & Mancur Olson. 1993. “Dictatorship, Democracy, and Development." 87(Sep.): 567-76. & 390 & 250 \\
\hline 14 & Arend Lijphart. 1971. "Comparative Politics and the Comparative Method." 65(Sep.): 682-93. & 346 & 270 \\
\hline 15 & $\begin{array}{l}\text { Nelson W. Polsby. 1968. "The Institutionalization of the U.S. House of Representatives." 62(Mar.): 144- } \\
68 .\end{array}$ & 324 & 279 \\
\hline 16 & Jonathan Bendor, Thomas H. Hammond. 1992. "Rethinking Allison's Models." 86(Jun.): 301-22. & 344 & 254 \\
\hline 17 & Peter Bachrach, Morton S. Baratz. 1962. "Two Faces of Power." 56(Dec.): 947-52. & 301 & 273 \\
\hline 18 & $\begin{array}{l}\text { Tracey E. George, Lee Epstein. 1992. "On the Nature of Supreme Court Decision Making." 86(Jun.): } \\
\text { 323-37. }\end{array}$ & 347 & 206 \\
\hline 19 & $\begin{array}{l}\text { Edward N. Muller, Mitchell A. Seligson. 1994. "Civic Culture and Democracy: The Question of Causal } \\
\text { Relationships." 88(Sep.): 635-52. }\end{array}$ & 341 & 191 \\
\hline 20 & $\begin{array}{l}\text { Richard F. Fenno Jr. 1977. "U.S. House Members in Their Constituencies: An Exploration." 71(Sep.): } \\
\text { 883-917. }\end{array}$ & 337 & 179 \\
\hline 21 & $\begin{array}{l}\text { Arthur H. Miller, Martin P. Wattenberg, Oksana Malanchuk. 1986. "Schematic Assessments of } \\
\text { Presidential Candidates." } 80 \text { (Jun.): 521-40. }\end{array}$ & 274 & 221 \\
\hline 22 & $\begin{array}{l}\text { William Mishler, Reginald S. Sheehan. 1993. "The Supreme Court as a Countermajoritarian Institution? } \\
\text { The Impact of Public Opinion on Supreme Court Decisions." 87(Mar.): 87-101. }\end{array}$ & 333 & 159 \\
\hline 23 & $\begin{array}{l}\text { Aaron Wildavsky. 1987. "Choosing Preferences by Constructing Institutions: A Cultural Theory of } \\
\text { Preference Formation." 81(Mar.): 3-22. }\end{array}$ & 313 & 176 \\
\hline 24 & $\begin{array}{l}\text { Anne Schneider, Helen Ingram. 1993. "Social Construction of Target Populations: Implications for } \\
\text { Politics and Policy." 87(Jun.): 334-47. }\end{array}$ & 278 & 208 \\
\hline 25 & Warren E. Miller, Donald E. Stokes. 1963. "Constituency Influence in Congress." 57(Mar.): 45-56. & 256 & 225 \\
\hline
\end{tabular}

Source: JSTOR (www.jstor.org).

Note: Ranking is by total viewings plus total printings. 